\title{
Radiofrequência na dor crônica
}

Radiofrequency in chronic pain

Radiofrecuencia en el dolor crónico

\author{
José Luciano Braun Filho' \\ Leandro Mamede Braun ${ }^{2}$
}

\section{RESUMO}

Radiofrequência (RF) é uma técnica minimamente invasiva alvo-seletiva e tem sido usada durante muitos anos para o tratamento de diferentes doenças, como dor lombar crônica, neuralgia trigeminal e outros. Trata-se de uma corrente elétrica alternada com frequência oscilatória de 500.000 hz, que flui através de um eletrodo introduzido percutaneamente. O calor é formado ao redor do eletrodo porque o tecido age como um resistor. Essa técnica pode, portanto, ser usada para causar lesões em tecidos nervosos no tratamento de dor crônica. O objetivo desta revisão é abordar alguns aspectos importantes do mecanismo e evolução da radiofrequência na dor crônica. Serão abordados os aspectos básicos da Física e o mecanismo de ação da radiofrequência, método que tem sido usado para tratar dores crônicas de diferentes etiologias, além da evolução com o advento da radiofrequência pulsátil. O uso da radiofrequência no manejo da dor crônica é uma ferramenta útil em diferentes condições dolorosas e tem sido usada com sucesso por mais de 25 anos. Seu mecanismo de ação ainda não foi totalmente elucidado. A radiofrequência pulsátil é, em particular, uma técnica minimamente destrutiva e poderá ser uma alternativa à forma convencional de tratamento para a

\section{ABSTRACT}

Radiofrequency $(R F)$ is a minimally invasive target-selective technique that has been used with success for many years in the treatment of different pathologies, like low back pain, trigeminal neuralgia and others. $R F$ is an alternating electric field with oscillation in the frequency of $500.000 \mathrm{~Hz}$ that flows through a percutaneously introduced electrode. The heat will be produced around the electrode, because the tissue acts as a resistor. Therefore, radiofrequency may be used to ablate nervous tissue in the treatment of chronic pain. The objective of this study was to review a fewimportant aspects of the mechanism and evolution of radiofrequency in chronic pain. The basic aspects of Physics and the mechanism of radiofrequency, a method which has been used to treat different etiologies of chronic pain, besides the evolution that came with the introduction of pulsed radiofrequency will be herein elucidated. The application of radiofrequency in the management of chronic pain may be a useful tool in the management of several chronic pain conditions and have been successfully used in clinical practice for more than 25 years. Its mechanism of action has not been totally elucidated yet. Pulsed radiofrequency, in private, is a minimally destructive procedure that

\section{RESUMEN}

Radiofrecuencia (RF) es una técnica mínimamente invasiva con blanco selectivo, y ha sido usada durante muchos años para el tratamiento de diferentes patologías como dolor lumbar crónico, neuralgia trigeminal entre otros. Radiofrecuencia es una corriente eléctrica alternada con frecuencia oscilatoria de $500.000 \mathrm{hz}$. La corriente fluye por medio de un electrodo introducido percutáneamente y el calor es formado alrededor del electrodo, porque el tejido actúa como una resistencia. Por lo tanto, la radiofrecuencia puede ser usada para causar lesiones en tejidos nerviosos para tratamiento de dolor crónico. El objetivo de esta revisión es abordar algunos aspectos importantes del mecanismo y evolución de la radiofrecuencia en el dolor crónico. Serán abordados los aspectos básicos de la fisica y el mecanismo de acción de la RF, método que ha sido usado para tratar dolores crónicos de diferentes etiologías, además de la evolución con el advenimiento de la radiofrecuencia pulsátil.El uso de la radiofrecuencia en el manejo del dolor crónico es una herramienta útil en diferentes condiciones dolorosas y ha sido usado con suceso por más de 25 años. Su mecanismo de acción aun no está totalmente elucidado. La radiofrecuencia pulsátil es en particular una técnica mínimamente destructiva y podrá ser una alternativa

\footnotetext{
Médico anestesiologista da World Institute of Pain; Especialista em Dor - Recife (PE), Brasil.

${ }^{2}$ Fellow of Intervention Pain Practice at World Institute of Pain; Médico assistente do Hospital das Clínicas da Faculdade de Medicina da Universidade Federal de Pernambuco - UFPE - Recife (PE), Brasil.
} 
dor por radiofrequência.

DESCRITORES: Dor/terapia;

Doença crônica; Ondas de rádio/uso terapêutico may offer alternatives for the pain treatment with radiofrequency. a la forma convencional para el tratamiento de dolor por radiofrecuencia.
KEYWORDS: Pain/therapy;

Chronic disease; Radio waves/therapeutic use
DESCRIPTORES: Dolor/terapia; Enfermedad crónica; Ondas radioeléctricas/uso terapéutico

\section{ASPECTOS HISTÓRICOS}

$\mathrm{O}$ uso de lesões por radiofrequência (RF) no tratamento da dor surgiu com o advento da cordotomia cervical lateral percutânea para dor oncológica unilateral ${ }^{1}$. Inicialmente, Mullan utilizou-se da técnica com corrente direta para a realização da cordotomia, mais tarde modificada por Rosomoff, que fez uso então da corrente por RF para produzir lesões mais circunscritas ${ }^{2}$. Poucos anos depois, Sweet desenvolveu sua técnica com RF no gânglio de Gasser (rizotomia trigeminal) para o tratamento da neuralgia trigeminal ${ }^{3}$.

O emprego da RF no tratamento da dor de origem espinhal iniciou-se com Shealy, em 1975, que realizava a lesão do ramo medial para dor de origem facetária na região lombar ou cervical ${ }^{4}$. Na ocasião, ele lançou mão de um eletrodo com diâmetro de 14 GAUGE $(\mathrm{G})$ introduzido por meio de uma agulha $12 \mathrm{G}$, um instrumento de calibre muito grosseiro em comparação aos mais modernos da atualidade.

Os anos 1980 foram um marco, quando eletrodos de pequeno diâmetro passaram a servir no tratamento da dor de origem espinhal ${ }^{5}$. O sistema SMK desenvolvido naquela ocasião consiste em uma cânula de calibre $22 \mathrm{G}$, acoplada a um fino eletrodo inserido nessa cânula. Isso permitiu lesões mais circunscritas e tornou o procedimento bem menos doloroso. Assim, tornou-se possível a realização de lesões no compartimento anterior (gânglio da raiz dorsal, ramo comunicante, cadeia simpática) com maior precisão e menor risco de lesões por desaferentação ou problemas de danos a fibras motoras secundários a grandes lesões ${ }^{6-8}$.

O desenvolvimento final se deu com o advento da aplicação da radiofrequência intradiscal para o tratamento da dor de origem discogênica. Desde a sua introdução, equipamentos mais sofisticados têm sido desenvolvidos para atender a esse propósito. Embora o mecanismo de ação ainda não esteja totalmente claro, os resultados são encorajadores ${ }^{9-12}$.

\section{ASPECTOS FÍSICOS DA RADIOFREQUÊNCIA}

A radiofrequência é um método de lesão por corrente elétrica alternada de alta frequência $(500.000 \mathrm{~Hz})$. A radiofrequência é aplicada através de um eletrodo de calibre $27 \mathrm{G}$, o qual é inserido através de agulha especial $22 \mathrm{G}$, exposto em sua parte mais distal. A parte metálica exposta não isolada distalmente é chamada de ponta ativa, e seu com- primento pode variar de 2 a $15 \mathrm{~mm}$. A corrente elétrica é produzida por um gerador especializado através de seus terminais (Figura 1). Conecta-se o paciente a uma placa dispersiva, a qual levará a corrente de volta ao gerador de RF; dessa forma, a quantidade de corrente que entra no corpo do paciente através do eletrodo de lesão deve ser igual à corrente que o deixará através da placa dispersiva (Figura 2). Assim, o corpo do paciente funciona como um dos elementos do circuito elétrico.

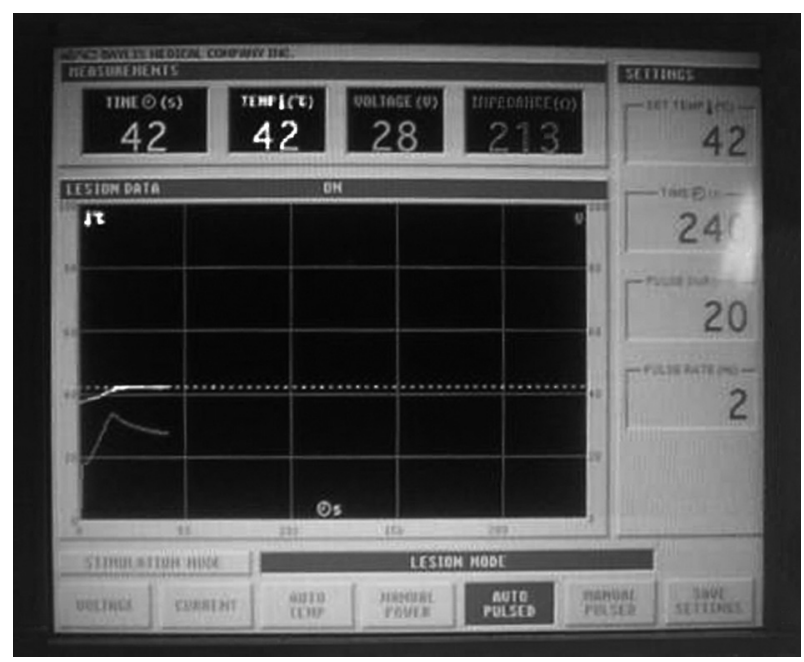

Figura 1

Gerador de radiofrequência.Bayles.

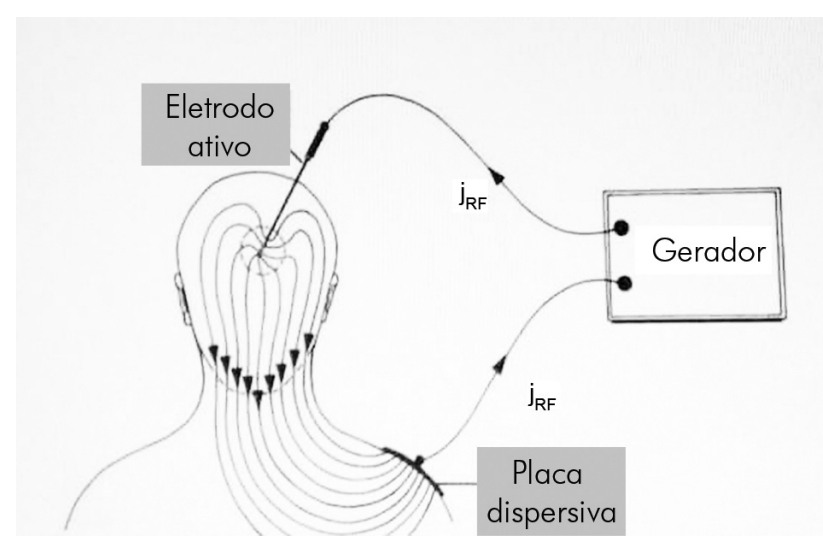

Figura 2

A propagação da densidade da corrente (jif) de radiofrequência no tecido entre ponta ativa e a placa dispersiva 
A área de superfície do terminal dispersivo é, muitas vezes, maior do que a área da ponta ativa, fazendo com que a densidade da corrente elétrica e voltagem se concentrem ao redor da ponta ativa. Forma-se calor pela passagem da corrente de radiofrequência através dos tecidos que, portanto, agem como um resistor, e a passagem da corrente elétrica pelo corpo produzirão calor. $\mathrm{O}$ tecido aquecerá e transmitirá secundariamente o calor ao eletrodo, não o inverso. O mecanismo de aquecimento é, portanto, iônico, ao invés de dielétrico.

Se o eletrodo apresentar um design correto e estiver adequadamente posicionado, sua ponta ativa não absorverá muito calor em estado de equilíbrio; dessa forma, a temperatura da ponta ativa será aproximadamente a área de maior calor tissular. Para a realização de uma lesão segura e de tamanho previsível, é de fundamental importância a escolha de um eletrodo de ponta ativa adequada e ter um controle rígido da temperatura de lesão.

A formação de calor será maior onde houver maior densidade de corrente elétrica, ou seja, ao redor da ponta do eletrodo e onde estará o tecido alvo de lesão por radiofrequência. A corrente se dispersa de maneira uniforme a partir do início da área não isolada, alcançando maior densidade na porção proximal na qual há uma área maior para saída da corrente. Desse modo, a lesão adquirirá uma conformação em forma de pêra com sua base ao redor da porção proximal da ponta ativa e muito pouca lesão adiante da porção distal (Figura 3). Como a ponta ativa do eletrodo promoverá mais lesões transversalmente, recomenda-se posicionar o eletrodo paralelamente ao tecido alvo, com o objetivo de aperfeiçoar a lesão térmica ao se utilizar a radiofrequência contínua convenciona ${ }^{13}$.

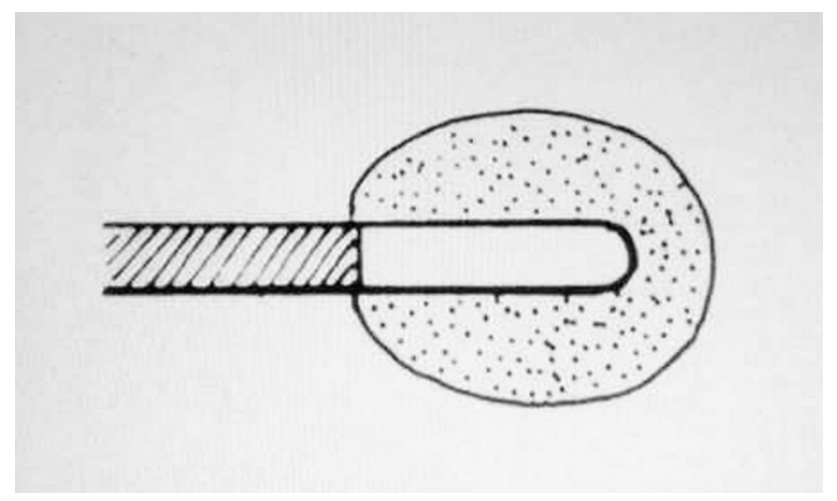

Figura 3

Configuração da lesão térmica. a lesão térmica apresenta forma de pera com a base na parte proximal da ponta ativa. a formação de calor adiante da ponta ativa e mínima.

A relação entre o gerador de radiofrequência e a temperatura atingida na ponta do eletrodo não se faz de forma direta. A temperatura final será determinada por variáveis relativas à sua produção e por sua dissipação. Um fator importante na produção de calor é a impedância (resistência à corrente alternada), que varia de acordo com os diferentes tecidos corporais. Essa produção oscila de forma diretamente proporcional à impedância. Outra variável relevante é a dissipação ou eliminação do calor, que depende de dois fatores principais: a condutividade do tecido e a sua vascularização, pois o fluxo sanguíneo dissipa o calor da área aquecida. Como ambos variam muito, a temperatura deve ser medida para se saber o resultado final entre a formação de calor e sua dissipação ${ }^{14}$.

A lesão térmica por radiofrequência tem duas fases: durante a primeira, é necessária uma grande produção de energia para elevação inicial da temperatura. Quanto menor a impedância, maior potência de corrente elétrica deverá ser gerada. A voltagem, nesta fase, oscila entre 25 e $60 \mathrm{~V}$. Ao se alcançar a temperatura desejada, inicia-se a segunda fase, cuja carga elétrica do gerador é gradualmente diminuída a um nível de equilíbrio para compensar a dissipação de calor ${ }^{14}$.

\section{EXPOSIÇÃO AO CAMPO ELÉTRICO}

O campo elétrico merece destaque especial e não apresenta relação com a corrente e com a formação de calor. A distribuição do campo elétrico difere do padrão de lesão térmica discutido anteriormente. A direção e a magnitude do campo elétrico são muito dependentes da conformação do condutor (ponta do eletrodo). Se houver conformação plana, o campo elétrico gerado será fraco. Se tiver formato circular, a carga elétrica será inversamente proporcional ao raio do círculo. Caso o condutor seja pontiagudo, formará um campo elétrico de maior magnitude, como observado com os eletrodos de ponta ativa pontiaguda. Isso significa que, ao redor da sua parte plana proximal da ponta descoberta, o campo elétrico será fraco, diferente do campo elétrico de maior intensidade gerado a partir da porção terminal e pontiaguda da ponta ativa $^{15}$ (Figura 4).

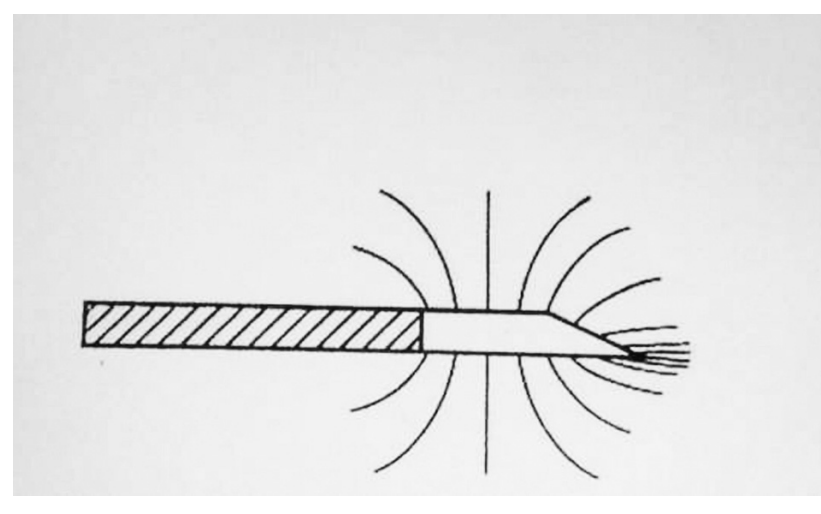

Figura 4

Formação do campo elétrico em relação a ponta ativa do eletrodo

Como podemos observar, os efeitos da RF têm duas ações distintas em sua distribuição espacial. A produção de calor se faz máxima em sua porção paralela à ponta ativa do eletrodo, com mínima produção de calor adiante da sua ponta distal. Por outro lado, o campo elétrico se comporta de forma oposta, com formação máxima distal à ponta ativa do eletrodo ${ }^{16}$.

Essa conformação espacial do campo elétrico produz implicações práticas óbvias, já que, se objetivarmos expor o 
tecido-alvo a um campo elétrico de maior intensidade, o eletrodo deverá ser posicionado perpendicularmente ao alvo e não paralelamente, como se faz para a exposição ao calor ${ }^{13}$.

\section{MECANISMO DE AÇÃO RADIOFREQUÊNCIA}

O mecanismo de ação da RF ainda é motivo de discussão e controvérsia. O papel do calor e seus efeitos clínicos nas lesões por radiofrequência não foram, no entanto, questionados durante anos. Desde o início, essa foi considerada uma técnica essencialmente neuroablativa. Várias observações clínicas não puderam ser explicadas apenas pela lesão térmica. Desde então, o papel da ação do campo elétrico magnético foi imputado na obtenção de alguns resultados independentes da temperatura e, portanto, o mecanismo de ação da radiofrequência tem sido repensado ${ }^{14-16}$.

Os efeitos do calor sobre os tecidos neurais são claramente destrutivos ${ }^{17}$. As alterações teciduais geralmente são reversíveis quando a temperatura chega aos $45^{\circ} \mathrm{C}$, sendo que a coagulação das proteínas ocorre em temperaturas acima de $60^{\circ} \mathrm{C}$. Uma vez que existe um gradiente de temperatura entre o centro da lesão e sua periferia, sendo essa última área exposta a temperaturas mais moderadas, algumas técnicas de RF deveriam objetivar expor os alvos a temperaturas mais moderadas no intuito de prevenir uma lesão por desaferentação. Nessas temperaturas, haveria um efeito seletivo em fibras não mielinizadas, segundo estudo realizado experimentalmente em gatos $^{18}$. Tais achados de seletividade sobre as fibras não mielinizadas e preservação das fibras de grande calibre mielinizadas não foram reproduzidos por diferentes pesquisadores em diversos estudos ${ }^{19,20}$. O conceito de que $o$ mecanismo de ação por meio do calor é puramente neuroablativo é muito simplista. Historicamente, os métodos ablativos no tratamento de dor têm se mostrado desapontadores. Outro fator importante é que, na maioria dos procedimentos de RF, o eletrodo é posicionado perpendicularmente ao alvo, o que não sustentaria o mecanismo baseado apenas no calor. Devido às controvérsias laboratoriais, experimentais e clínicas, outros mecanismos além do calor devem estar envolvidos.

A formação de calor não é a única ocorrência ao se aplicar RF nos tecidos. O tecido que circunda o eletrodo é também exposto ao campo elétrico da RF, como dito anteriormente. A questão reside em definir se isto tem algum efeito biológico independente da temperatura. O campo elétrico gerado por RF é usado por biólogos para separar diferentes tipos celulares em cultura. Archer, estudando se essa exposição teria algum efeito biológico, não observou diferenças na morfologia, taxa mitótica ou respiração, mas encontrou alteração na expressão de genes C-Fos e outros genes intermediários ${ }^{21}$. Ao se aplicar RFP no gânglio da raiz dorsal de ratos, o gene C-Fos é expresso nas lâminas I e II correspondentes ${ }^{22}$. Adicionalmente, observaram-se modulação de sinapses excitatórias em neurônios de tecido nervoso de hipocampo orgânico submetido à RFP ${ }^{23}$.

\section{RADIOFREQUÊNCIA PULSÁTIL}

Com essa linha de raciocínio, a próxima etapa de estudos seria encontrar um método de aplicação da RF sem elevar a temperatura a níveis neuroablativos, considerando-se, então, que um novo mecanismo de ação estaria atuando ${ }^{24,25}$.

Tal objetivo poderia ser alcançado basicamente de três maneiras: a) pelo resfriamento da ponta do eletrodo com solução salina resfriada, porém poderia gerar altas temperaturas na ponta do eletrodo; b) pela diminuição da corrente gerada, mas isso só seria possível com o uso de campos elétricos fracos; c) pela interrupção da corrente e consequente diminuição da temperatura pela condutividade térmica e circulação próxima da área aplicada, diminuindo assim a temperatura. Esse último método, aplicação de RF, foi então denominado radiofrequência pulsátil (RFP). Nessa técnica, a corrente de saída do gerador é interrompida por períodos de pausa. A forma usual é de 2 ciclos de 20 milisegundos (ms) por cada ciclo ativo (Figura 5).

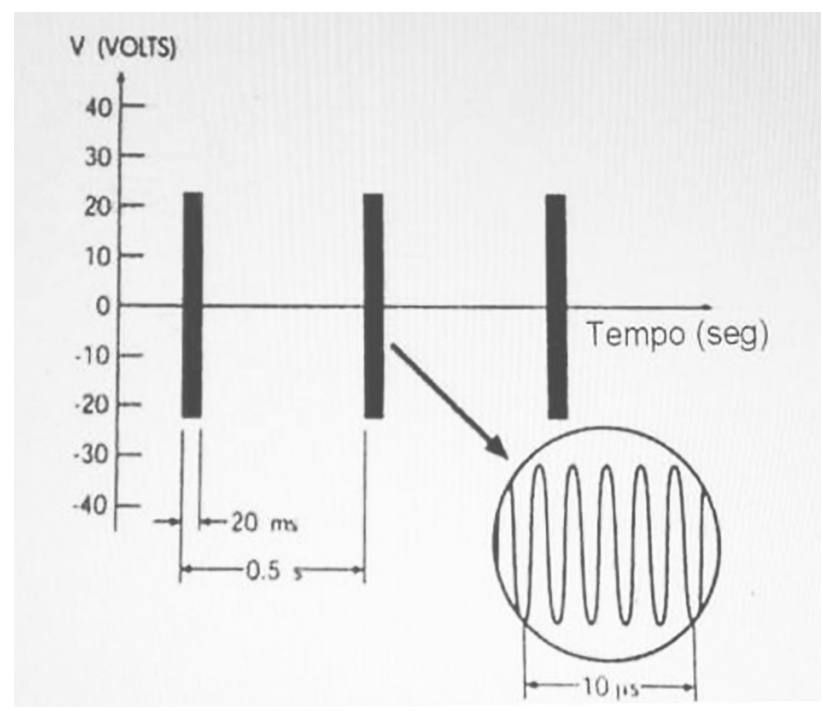

Figura 5

Esquema do ciclo típico de radiofrequência pulsatil. Há dois ciclos por segundo de 20 milisegundos cada

Os resultados do emprego da RFP revelam-se encorajadores $^{26-28}$. Por seu caráter modulatório e não ablativo, a RFP tem sido usada em regiões antes não aprovadas, como gânglio da raiz dorsa ${ }^{29}$, nervos periféricos ${ }^{28}$, cadeia simpática, e até pontos gatilhos, porém se fazem necessários mais estudos que abordem o tema, pois se trata de um método novo.

\section{EQUIPAMENTO DE RADIOFREQUÊNCIA}

$O$ gerador moderno de radiofrequência inclui não apenas o meio pelo qual será realizada a lesão e monitorizada a temperatura, mas também possui dispositivos auxiliares que melhoram a acurácia e segurança do procedimento.

O aparelho moderno é uma máquina com microprocessador de várias funções que permitem um acompanhamento e geração da lesão, além de um monitor com leitor 
avançado de vários parâmetros extremamente importantes, como impedância, parâmetros de estimulação sensorial e motor, voltagem, corrente, temperatura etc. Como o sistema é baseado em um microprocessador, múltiplas funções podem ser monitoradas simultaneamente. $\mathrm{O}$ seu monitoramento de display alfanumérico permite ao operador avaliar em tempo real os diversos parâmetros ${ }^{30}$. Tradicionalmente, é de boa prática uma operabilidade formal dos seguintes parâmetros ao se realizar a lesão:

- Impedância: é uma medida essencial na detecção de curtos circuitos ou quebras, e de suma importância na detecção de diferentes impedâncias em diferente tecidos. Por exemplo, ao se fazer um procedimento fiscal, uma quebra da impedância traduz a entrada da agulha no núcleo pulposo ao invés do anel fibroso, onde ela é muito maior.

- Estímulos elétricos: geralmente utilizam-se estímulos com correntes a $50 \mathrm{hz}$ para estimulação sensitiva e a 2 hz para estimulação motora. A realização desses testes é significativa para confirmação da posição do eletrodo em relação à estrutura alvo. A estimulação sensorial confirma a proximidade do alvo; a motora, a distância segura das fibras motoras, essencial ao se causarem lesões térmicas.
- Temperatura: o controle da temperatura é de vital importância e já foi explicada. Durante o uso de RFP, a temperatura deve ser medida para evitar níveis neuroablativos. Ocasionalmente, a dissipação do calor é lenta e pequenos ajustes no gerador serão necessários durante o procedimento.

\section{CONCLUSÃO}

O tratamento com RF vem se ampliando, sendo muito utilizado nos últimos anos. Constitui-se de procedimentos que ocorrem, na maioria das vezes, de forma minimamente invasiva, com alvos seletivos, geralmente feitos em regime ambulatorial e com incidência muito baixa de complicações e efeitos adversos se realizada por profissionais capacitados e devidamente treinados.

Sua indicação justifica-se em pacientes refratários ao tratamento conservador e como uma alternativa a outros mais invasivos e onerosos. Os resultados dos estudos têm variado devido à discrepância dos protocolos, do critério de seleção de pacientes e da avaliação final, o que torna uma metanálise difícil.

O desenvolvimento da radiofrequência pulsátil tem ampliado novas perspectivas para o tratamento com RF.

\section{REFERÊNCIAS}

1. Mullan S, Hekmatpanah J, Dobben G, Beckman F. Percutaneous, intramedullary cordotomy utilizing the unipolar anodal electrolytic lesion. J Neurosurg. 1965;22(6):548-53.

2. Rosomoff HL, Brown CJ, Sheptak P. Percutaneous radiofrequency cervical cordotomy: technique. J Neurosurg. 1965;23(6):639-44.

3. Sweet WH, Wepsic JG. Controlled thermocoagulation of trigeminal ganglion and rootlets for differential destruction of pain fibers. 1 . Trigeminal neuralgia. J Neurosurg. 1974;40(2):143-56.

4. Shealy CN. Percutaneous radiofrequency denervation of spinal facets. Treatment for chronic back pain and sciatica. J Neurosurg. 1975;43(4):448-51.

5. Sluijter ME, Mehta M. Treatment of chronic back and neck pain by percutaneous thermal lesion. In: Lipton S, Miles J, editors. Persistent pain: modern methods of treatment. London; New York: Academic Press: Grune \& Stratton; 1977-[c1985]. London: Academic Press; 1981. v. 3, p. 141-79.
6. Lord SM, Barnsley L, Wallis BJ, McDonald GJ, Bogduk N. Percutaneous radio-frequency neurotomy for chronic cervical zygapophyseal-joint pain. N Engl J Med. 1996;335(23):1721-6. Comment in: ACP J Club. 1997;126(3):71. N Engl J Med. 1996; 335(23):1763-4. N Engl J Med. 1997;336(21):1530-1.

7. Sluijter ME. The use of radiofrequency lesions for pain relief in failed back patients. Int Disabil Stud. 1988;10(1):37-43.

8. Pernak J. Percutaneous radiofrequency thermal lumbar sympathectomy and its clinical use. Delft, Holland: Eburon; 1988.

9. Van Kleef M, Barendse GAM, Wilmink JT. Percutaneous intradiscal radiofrequency thermocoagulation in chronic non-specific low back pain. Pain Clin. 1996;9:259-68.

10.Saal JA, Saal JS. Intradiscal electrothermal treatment for chronic discogenic low back pain: a prospective outcome study with minimum 1-year follow-up. Spine. 2000;25(20):2622-7.

11. Wright RE. Discogenic pain. In: Sluijter ME, editor. Radiofrequency: Part 1. Meggen, Switzerland: Flivo Press; 2001. p. 149-69.
12.Karasek M, Bogduk N. Twelvemonth follow-up of a controlled trial of intradiscal thermal anuloplasty for back pain due to internal disc disruption. Spine. 2000;25(20):2601-7.

13.Bogduk N, Macintosh J, Marsland A. Technical limitations to the efficacy of radiofrequency neurotomy for spinal pain. Neurosurgery. 1987;20(4):529-35.

14.Sluijter ME, Racz G. Technical aspects of radiofrequency. Pain Pract. 2002;2(3):195-200.

15.Halliday D, Resnick R. The electric field. In: Halliday D, Resnick R. Physic. 3rd ed. New York: John Wiley \& Sons; 1978. Pt. 2, p. 580-600.

16. Sluijter ME. Some specific pain syndromes. In: Sluijter ME, editor. Radiofrequency: part 1. Meggen, Switzerland: Flivo Press; 2001. p. 176-7.

17.Van Kleef M. Radiofrequency lesions adjacent to the dorsal root ganglion. Thesis. Maastricht: Datawyse/ University Press; 1996.

18.Letcher FS, Goldring S. The effect of radiofrequency current and heat on peripheral nerve action potential in the cat. J Neurosurg. 1968;29(1):42-7. 
19.Louw AJ, VIes HS, Freling G, Herpers MJ, Arends JW, Van Kleef M. The morphological effects of a radio frequency lesion adjacent to the dorsal root ganglion (RP-DRG): an experimental study in the goat. Eur J Pain. 2001;5(2):169-74.

20.Smith HP, McWorther JM, Challa VZ. Radiofrequency neurolysis in a clinical model. Neuropathological correlation. J Neurosurg. 1981;55(2):246-53.

21.Archer S, Li TT, Evans AT, Britland ST, Morgan H. Cell reactions to dielectrophoretic manipulation. Biochem Biophys Res Commun. 1999;257(3):687-98.

22.Higuchi Y, Nashold BS Jr, Sluijter M, Cosman E, Pearlstein RD.

Exposure of the dorsal root ganglion in rats to pulsed radiofrequency currents activates dorsal horn lamina I and II neurons. Neurosurgery. 2002;50(4):850-5; discussion 856.

23. Cahana A, Muller D. PRF but not RF lesions, transiently modulate excitatory synaptic transmission in organotypic nervous tissue cultures. Pain. 2002;3(Suppl 1):25.
24.Sluijter ME, Cosman E, Rittman W, van Kleef M. The effect of pulsed radiofrequency fields applied to the dorsal root ganglion: a preliminary report. Pain Clin. 1998;11:109-17.

25.Sluijter ME, Van Kleef M. Characteristics and mode of action of radiofrequency lesions. Curr Pain Head Rep. 1998;2(3):143-50.

26.Munglani R. The longer term effect of pulsed radiofrequency for neuropathic pain. Pain. 1999;80(1-2):437-9.

27.Erdine S, Talu GK. Percutaneous rhizotomy application with pulseRFTC in FBSS: case report [Abstract]. Worldwide Conference on Pain. San Francisco; 2000.

28.van Kleef M, Spaans F, Dingemans W, Barendse GA, Floor E, Sluijter ME. Effects and side effects of a percutaneous thermal lesion of the dorsal root ganglion in patients with cervical pain syndrome. Pain. 1993;52(1):49-53.
29.Van Zundert J, Patijn J, Kessels A, Lamé I, van Suijlekom H, van Kleef M. Pulsed radiofrequency adjacent to the cervical dorsal root ganglion in chronic cervical radicular pain: a double blind sham controlled randomized clinical trial. Pain. 2007;127(1-2):173-82. Comment in: Expert Rev Neurother. 2007;7(5):4712. Pain. 2007;127(1-2):3-4.

30.Kline MT. Radiofrequency techniques in clinical practice. In: Waldman SD, Winnie AP, editors. Interventional pain management. Philadelphia: W.B. Saunders; 1996. p. 185-217.

\section{Correspondência}

José Luciano Braun Filho

Rua Baltazar Pereira, 70/501

CEP: $51011-550$ - Recife (PE), Brasil

Tel.: (81) 9974-7471

E-mail: Ibraun@terra.com.br 